\title{
Der akute Abdominalschmerz in der Notfallambulanz - ein klinischer Algorithmus für den erwachsenen Patienten
}

\author{
Acute Abdominal Pain in the Emergency Department - \\ A Clinical Algorithm for Adult Patients
}

Autoren

Institute
H. Trentzsch ${ }^{1}$, J. Werner ${ }^{2}$, K.-W. Jauch ${ }^{1}$

Klinikum der Universität München Campus Großhadern, Chirurgische Klinik und Poliklinik, München, Deutschland

Klinikum der Universität München Campus Innenstadt, Chirurgische Klinik, München, Deutschland

\section{Schlüsselwörter \\ D Abdominalchirurgie \\ - abdomineller Notfalleingriff \\ akuter Bauch \\ - Algorithmus \\ D akuter Abdominalschmerz \\ D Workflow \\ Key words \\ D abdominal surgery \\ emergency abdominal ope- rations \\ - acute abdomen \\ - algorithm \\ - acute abdominal pain \\ work flow}

\section{Zusammenfassung}

$\nabla$

Hinter plötzlich auftretendem Abdominalschmerz steht ein umfangreicher Symptomkomplex, zu dem auch eine Vielzahl von chirurgisch zu behandelnden Ursachen gezählt wird. Dieser Symptomkomplex repräsentiert den häufigsten chirurgischen Notfall, den häufigsten Grund für eine chirurgische Konsultation in der Notfallambulanz und den häufigsten Grund für eine nichtunfallbedingte stationäre Aufnahme. Nicht selten ist eine operative Intervention zur Behandlung der zugrunde liegenden Ursache erforderlich. Die goldene Handlungsmaxime besteht darin, schnell zu erkennen, ob die Ursache eine dringliche oder gar sofortige Indikation zur operativen Intervention darstellt. Es zeigt sich aber, dass sich hinter dem identischen Leitsymptom auch harmlose oder weniger dringliche Probleme als Ursache verbergen können. Die Schwierigkeit besteht darin, bei kosteneffizientem Einsatz verschiedener diagnostischer Maßnahmen und unter Wahrung des Strahlenschutzes diejenigen Patienten mit einer Notwendigkeit zur dinglichen operativen Therapie von denen zu trennen, die bei weniger gravierenden Ursachen konservativ und ohne Zeitdruck behandelt werden können. Der Umgang mit einem solchen hochkomplexen Entscheidungsprozess legt die Forderung nach einem klinischen Algorithmus nahe. Zahlreiche Publikationen haben in diesem Zusammenhang verschiedene Aspekte der initialen Beurteilung und dem Management dieses Symptomkomplexes wissenschaftlich untersucht. Interessanter Weise finden sich im Prinzip bisher aber keine Arbeiten, die entsprechend der formalen Anforderungen eine prioritäts- und problemorientierte Herangehensweise beschreiben. Klinische Algorithmen dienen dazu, den Umgang mit komplexen Krankheitsbildern wie dem Symptomkomplex des akuten Bauchschmerzes in einen übersichtlichen, logisch koordinierten und systematischen Ge-

\section{Abstract \\ $\nabla$}

Acute abdominal pain represents the cardinal symptom behind a vast number of possible underlying causes including several ones that require surgical treatment. It is the most common surgical emergency, the most common cause for a surgical consultation in the emergency department and the most common cause for non-trauma related hospital admissions. The golden mission statement is to rapidly identify whether the underlying cause requires an urgent or even immediate surgical intervention. However, behind the same cardinal symptom one may encounter harmless or non-urgent problems. By employing diagnostic means cost effectively and with the aim to avoid unnecessary exposure of the patient to X-rays in mind, the challenge remains to identify patients with an indication for emergency surgery from those who suffer from a less serious condition and thus can be treated conservatively and without any pressure of time. Dealing with such a highly complex decision-making process calls for a clinical algorithm. Many publications are available that have scrutinised the different aspects of the initial assessment and the emergency management of acute abdominal pain. However, the large body of evidence seems to miss articles that describe a formally correct priority- and problem-based approach. Clinical algorithms apply to complex disease states such as acute abdominal pain and translate them into one clearly laid out, logically coordinated and systematic overall process. Our intention is to develop such an algorithm to approach acute abdominal pain from the surgeon's point of view. Based on daily practice and with reference to available literature, it is the aim of this study to define a work flow that simply summarises all steps involved and defines the required decision process in order to form the intellectual basis for an evidence-based clinical algorithm. The result is illus- 
samtprozess zu übersetzen. Unser Anliegen ist es, einen klinischen Algorithmus zum akuten Abdominalschmerz aus der chirurgischen Perspektive zu entwickeln. Das Ziel dieser Arbeit ist es, in Anlehnung an die täglich geübte Praxis und unter Berücksichtigung der verfügbaren Evidenz einen Workflow zu formulieren, der neben einer schlichten Aneinanderreihung der Einzelprozesse auch die zu treffenden Entscheidungen zusammenstellt, um die intellektuellen Rahmenbedingungen zur Schaffung eines evidenzbasierten, klinischen Algorithmus aufzustellen. Das Ergebnis zeigt den ersten Schritt für die Erstellung eines solchen evidenzbasierten Algorithmus zur Evaluierung von erwachsenen Patienten mit akutem Abdominalschmerz. trated as a first draft of such an evidence-based algorithm to allow emergency evaluation of adult patients with acute abdominal pain.

\section{Einleitung \\ $\nabla$}

Das akute Abdomen ist definiert als plötzlich einsetzende, abdominale Schmerzen mit kurzem zeitlichen Verlauf, nicht länger als eine Woche [1]. Zwischen 5-10\% aller Patienten, die die Notfallambulanz aufsuchen, kommen wegen akuter Abdominalschmerzen. Das akute Abdomen stellt damit den häufigsten chirurgischen Notfall, den häufigsten Grund für eine chirurgische Konsultation in der Notfallambulanz und den häufigsten Grund für eine nicht-unfallbedingte stationäre Aufnahme dar [2]. Nicht selten ist eine operative Intervention zur Behandlung der zugrundeliegenden Ursache erforderlich. Es liegen zahlreiche epidemiologische Studien vor, die relativ homogen die häufigsten Ursachen beschreiben. Die mit über 10000 Patienten wohl repräsentativste Studie ist die der World Organization of Gastroenerology (OMGE). Sie weist als häufigste Diagnose mit 34\% den nicht-spezifischen Abdominalschmerz aus. Von den übrigen $66 \%$ fanden sich als Ursache in 75\% der Fälle die akute Appendizitis, die Dünndarmobstuktion und gynäkologische Ursachen [3].

Unter dem Leitsymptom „akuter Abdominalschmerz“ wird eine ganze Litanei auslösender Ursachen subsummiert, von denen eine Auswahl in D Tab. 1 aufgelistet ist und die in Übersichtsarbeiten wie z.B. von Lankisch [4] und Flasar [5] hervorragend beschrieben sind. Es zeigt sich, dass sich hinter dem identischen Leitsymptom auch harmlose oder weniger dringliche Probleme als Ursache verbergen können. Die Schwierigkeit besteht darin, die Notwendigkeit zur operativen Therapie mit höchster Priorität von weniger gravierenden Ursachen zu trennen, die konservativ und ohne Zeitdruck behandelt werden können. Nicht zu unterschätzen sind dabei Ursachen, die in die Zuständigkeit anderer Fachdisziplinen fallen und deshalb eine Evaluierung im interdisziplinären Schulterschluss erfordern oder gar extra-abdominalen Ursprungs sind. Aus chirurgischer Sicht ist die Unterscheidung in akutes Abdomen und unklaren Abdominalschmerz aus diesem Blickwinkel sinnvoll.

Für den Erstkontakt mit dem Patienten in der Notfallambulanz besteht die goldene Handlungsmaxime darin, schnell zu erkennen, ob die Ursache eine dringlich oder gar sofortige Indikation zur operativen Intervention darstellt [6]. Angesichts der Komplexität des gesamten Symptomkomplexes „akuter Bauchschmerz“ stellt sich die Frage nach problem- und prioritätenorientieren Behandlungsempfehlungen, die es auch dem unerfahrenen Anwender erlaubt, bei Konfrontation mit dem Leitsymptom „akuter Bauchschmerz" selbst unter Zeitdruck eine gezielte und reproduzierbare Entscheidung zur diagnostischen Evaluierung und Festlegung der Therapiepriorität vorzunehmen.

Einige Autoren haben sich mit dieser komplexen Thematik beschäftigt, die Form der Niederlegung ist üblicher Weise aber die als Prosatext formulierte Expertenmeinung. Herausragende Arbeiten, die in die Thematik tiefer eintauchen, sind die kürzlich erschienene Arbeit von Grundmann [7] oder der von Delcore erarbeitete Workflow aus den „Principles and Practices“ des American College of Surgeons [1]. Alle verfügbaren Arbeiten konzentrieren sich dabei jedoch auf fixierte logische Sequenzen einzelner Entscheidungsprozesse, ohne dabei zeitkritische Entscheidungen unter Berücksichtigung des Zustandes des Patienten vorzunehmen. Klinische Algorithmen, die solche Entscheidungsprozesse nach festen Regeln beschreiben, können hier eine Verbesserung bewirken [8].

Ziel der vorliegenden Arbeit ist es, festzustellen, in welchem Umfang algorithmusbasierte Behandlungsempfehlungen für den akuten Bauchschmerz bereits existieren und die notwendigen Schritte im Workflow der Evaluierung des akuten Bauchschmerzes beim Erwachsenen zu identifizieren, da es nur bei genauer Kenntnis der theoretischen und praktischen Grundlagen möglich ist, die Schlüsselfragen aufzudecken, die die Rahmenbedingungen für einen evidenzbasierten Algorithmus darstellen, der den Anforderungen der täglichen Praxis gerecht werden kann.

\section{Methodik \\ $\nabla$}

Wir führen in den Datenbanken MEDLINE, EMBASE und Cochrane eine nicht-systematische Literatursuche nach Arbeiten durch, die innerhalb der letzten 10 Jahre publiziert wurden. Für die Suche wurde das kontrollierte Vokabular der Medical Subject Heading (MeSH) verwendet. Die Suche nach „Algorithms“[Mesh] OR „work flow“ AND „Abdominal Pain“[Mesh] AND (Humans[Mesh]) lieferte 69 Arbeiten. Die Mehrzahl der Arbeiten befasste sich mit speziellen Diagnosen und wurde deshalb von uns nicht weiter berücksichtig, ebenso wie Leserbriefe, Editorials oder Artikel mit pädiatrischer Ausrichtung. Darüber hinaus wurden die Referenzen der Artikel im Sinne einer Handsuche nach weiteren relevanten Quellen durchgesehen.

Keiner der Beiträge, die sich mit dem gesamten Symptomkomplex akuter Abdominalschmerz befassen, beschreibt einen prioritäten- und problemorientierten klinische Algorithmus entsprechend den formalen Kriterien [8-10]. Als relevante Arbeiten im Bezug auf die Problemstellung wurden insgesamt 5 Arbeiten [1, $6,7,11,12$ ] ausgewählt, anhand derer ein Algorithmus in Anlehnung an die geübte Praxis in unserer Institution entwickelt wurde. Die systematische Anordnung der Entscheidungsknoten geschieht dabei prioritätenorientiert und legt so den Zeitpunkt und Ablauf der jeweiligen Einzelprozesse in einer logischen Reihenfolge fest [10]. Entsprechend den Vorgaben von Khalil et al. [9] soll der Algorithmus aus pragmatischen Gründen den Umfang 


\begin{tabular}{|c|c|c|}
\hline $\begin{array}{l}\text { rechter oberer Quadrant: } \\
\text { peptisches Ulkus }\end{array}$ & $\begin{array}{l}\text { epigastrisch: } \\
\text { peptisches Ulkus }\end{array}$ & $\begin{array}{l}\text { linker oberer Quadrant: } \\
\text { peptisches Ulkus }\end{array}$ \\
\hline Gallenkolik & Duodenitis & Milzinfarkt \\
\hline Cholezystitis & Pankreatitis & Milzruptur \\
\hline Choledocholithiasis & Gastroenteritis & Pankreatitis \\
\hline Cholangitis & mesenteriale Adenitis & Neoplasma des Pankreas \\
\hline Hepatitis & entzündliche Darmerkrankung & Pneumonie \\
\hline Neoplasma der Leber & mesenterialer Verschluss & Lungenembolie \\
\hline Leberabszess & & Pneumothorax \\
\hline Pneumonie & & subphrenischer Abszess \\
\hline Lungenembolie & & Pyelonephritis \\
\hline Pneumothorax & & Nephrolithiasis \\
\hline subphrenischer Abszess & & Kolitis \\
\hline Pyelonephritis & & myokardiale Ischämie \\
\hline Nephrolithiasis & & Herpes Zoster \\
\hline Kolitis & & \\
\hline myokardiale Ischämie & & \\
\hline Herpes Zoster & & \\
\hline & periumbilikal: & \\
\hline & Appendizitis (Frühphase) & \\
\hline & Dünndarmobstruktion & \\
\hline & Gastroenteritis & \\
\hline & BAA Ruptur & \\
\hline & Dissektion Aorta abdominalis & \\
\hline & mesenteriale Ischämie & \\
\hline & Hernie & \\
\hline rechter unterer Quadrant: & hypogastrisch: & linker unterer Quadrant: \\
\hline Appendizitis & entzündliche Darmerkrankung & Sigmadivertikulitits \\
\hline entzündliche Darmerkrankung & Ovarialtumor & entzündliche Darmerkrankung \\
\hline Ovarialtumor & Tuboovarialabszess & Ovarialtumor \\
\hline Tuboovarialabszess & stielgedrehte Ovarialzyste & Tuboovarialabszess \\
\hline stielgedrehte Ovarialzyste & Torsion des Ovars & stielgedrehte Ovarialzyste \\
\hline Torsion des Ovars & Salpingitis & Torsion des Ovars \\
\hline Salpingitis & ektope Schwangerschaft & Salpingitis \\
\hline ektope Schwangerschaft & Zystitis & ektope Schwangerschaft \\
\hline Hernie & Proktokolitis & Hernie \\
\hline Pyelonephritis & Divertikulitis & \\
\hline Nephrolithiasis & Prostatitis & \\
\hline & Endometriose & \\
\hline
\end{tabular}

Tab. 1 Differenzialdiagnosen des akuten Abdomens nach anatomischer Lokalisation der Schmerzen. Diese Übersicht erhebt keinen Anspruch auf Vollständigkeit. Die Tatsache, dass in den verschiedenen Lokalisationen dieselben Ursachen nebeneinander auftreten können, illustriert die Schwierigkeit, die richtige Diagnose zu stellen und daraus die richtige, $u$. $U$. zeitkritische Therapieentscheidung abzuleiten.

diffuse Schmerzen (ausgedehnt auf das gesamte Abdomen):

Peritonitis, Gastroenteritis, IBD, diabetische Ketoazidose, Opiatentzug, Porphyrie, Sichelzellkrise, Vaskulitis, Schwermetallintoxikation,

familiäres Mittelmeerfieber, intestinale Obstruktion

von einer Seite nicht überschreiten. Sequenzielle Entscheidungen wurden durch den Gebrauch von Checklisten abgekürzt. Je nach Entscheidung verzweigt sich der Workflow, wobei die festgestellte Probleme in Schleifen bearbeitet werden, die dann zum Workflow zurückführen. Auf dem Weg durch den Entscheidungsbaum führen die unterschiedlichen Befunde zu verschiedenen therapeutischen Endpunkten.

\section{Prioritäten-gesteuerter Workflow zur Beurteilung des akuten Abdominalschmerz \\ $\nabla$}

Der im Folgenden beschriebene Algorithmus ( $\boldsymbol{Q}$ Abb. 1) soll bei Patienten>18 Jahren mit der Verdachtsdiagnose „akutes Abdomen“, definiert als akut aufgetretener Bauchschmerz, der seit weniger als 1 Woche besteht, zur Anwendung kommen. Der Algorithmus ist erweitert um einen standardisierten Bogen, der sich an dem Vorschlag der OMGE orientiert, zur Erhebung von Anamnese und Befund ( $\triangle \mathbf{A b b} . \mathbf{2}$ ), wodurch eine transparente und vollständige Dokumentation von Untersuchungsbefund und der Entscheidungsbildung möglich ist.

\section{Anamnese und körperliche Untersuchung}

In Übereinstimmung mit sämtlichen Publikationen ist das wichtigste Hilfsmittel zur Evaluierung des akuten Abdomens eine detaillierte Anamnese und eine akkurate körperliche Untersuchung. Gerade bei typischer Klinik kann die Diagnose u.U. schon ohne Zuhilfenahme technischer Hilfsmittel erkannt werden. Beispielhaft seien "Augenscheindiagnosen“ wie eine inkarzerierte Hernie oder die typische akute Appendizitis mit periumbikal beginnendem, im Verlauf in den rechten Unterbauch wandernden Schmerz und Druckschmerz über dem McBurney Punkt genannt. Allein anhand der klinischen Bewertung kann die richtige Diagnose mit einer Sensitivität von $88 \%$ und einer Spezifität von $41 \%$ gestellt werden [13]. Anamnese und körperlicher Untersuchungsbefund sollten aufbauen auf ausreichender klinischer Erfahrung, genauer Kenntnis der Anatomie und Physiologie der Bauchhöhle und einer klaren Vorstellung der pathologischen Abläufe [5]. Es hat sich gezeigt, dass der Einsatz von standardisierten Bögen zur Anamnese und Befunderhebung deutlich zur Verbesserung der diagnostischen Treffsicherheit beiträgt [1]. Die - Abb. 2 zeigt einen solchen Bogen, der in den Algorithmus als "Standardbogen“ in den ersten Schritt implementiert ist (D Abb. 1): 

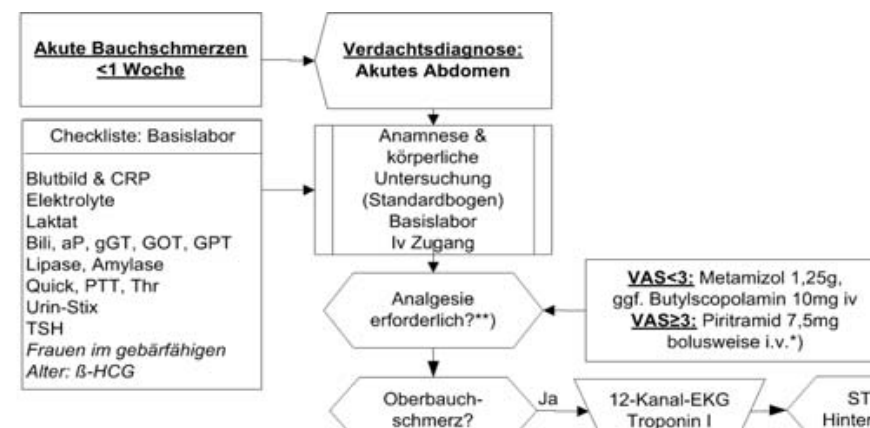

(2)

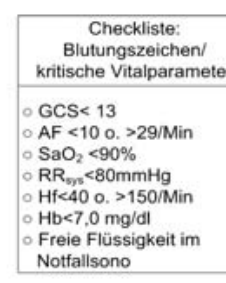

Checkliste: Obstruktion - zentraler, kolikartiger Schmerz

Distension

- Verstopfung

- Erbrechen

\section{Checkliste:
Peritoneale Zeichen} Peritoneale Zeichen
(Peritonismus)

Abwehrspannung

Loslassschmerz
Lowehrspannung

- Hustenschmerz
- Erschütterungsschmerz

\begin{tabular}{|c|}
\hline Checkliste \\
Generalisierte Peritonitis
\end{tabular}

Generalisierte Peritonitis diffuser Abd.-S

bretthartes Abdomen

Loslassschmerz

- Abwehrspannung

\begin{tabular}{|c|}
\hline $\begin{array}{l}\text { Checkliste: } \\
\text { Sepsiszeichen }\end{array}$ \\
\hline 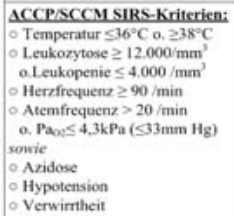 \\
\hline $\begin{array}{c}\text { Checkliste: } \\
\text { Ischämiezeichen: }\end{array}$ \\
\hline $\begin{array}{l}\text { - Fieber } \\
\text { - Tachykardie } \\
\text { - Hypotension } \\
\text { - Laktatanstieg }\end{array}$ \\
\hline
\end{tabular}

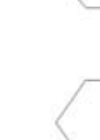

Nein
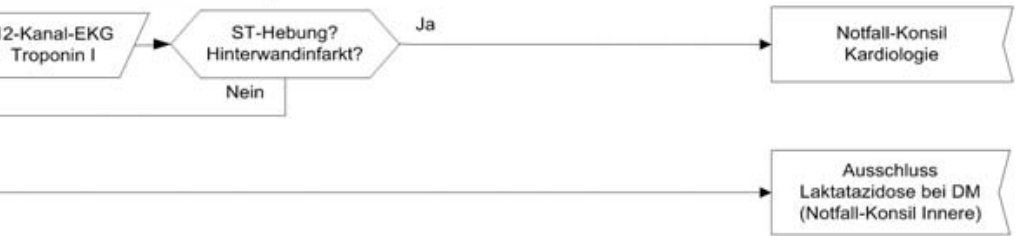

Basislabor:

BZ \& Laktat
erhöht?

Nein


Nein
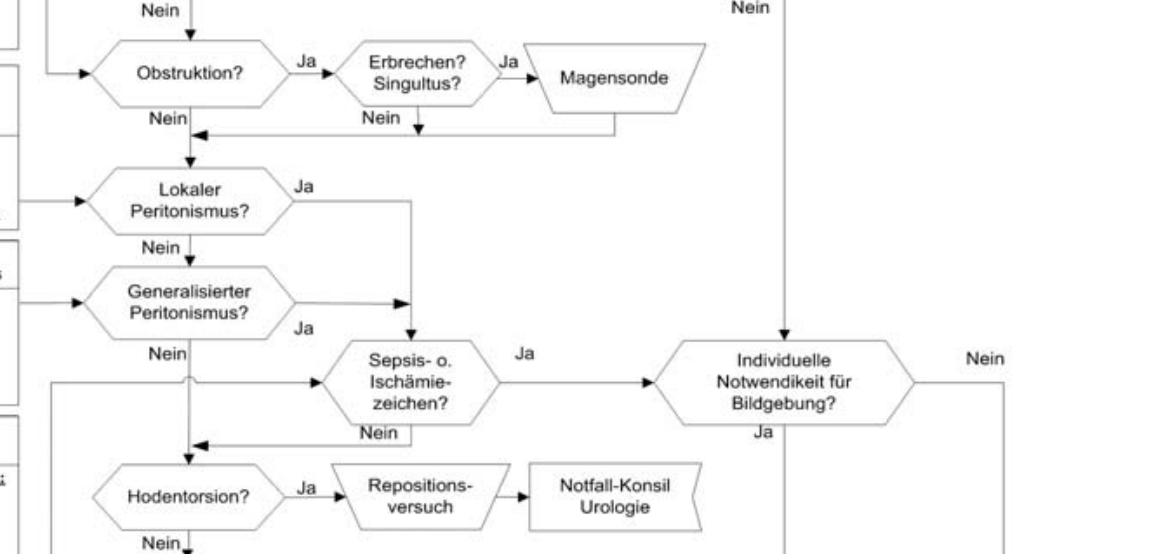

Nein

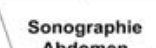

Abdomen

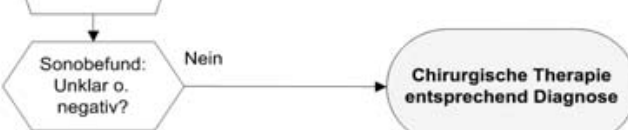

negativ?

Ja.

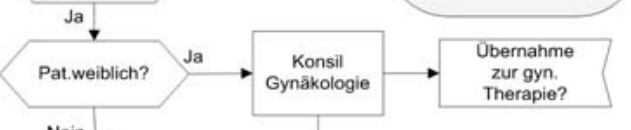

Checkliste:
Risikofaktoren

Schmerzen $<48$ i

Schmerz nach

Erbrechen

- Alter 265 Jahre

Voroperation


Auffalliges Labor o.

Untersuchungsbefund Ja

und Risikofaktoren für

.chir. Abdomen"?

\begin{tabular}{l} 
Nein \\
\hline v.a. nicht- \\
\hline
\end{tabular}

v.a. nicht-
spezifischen

Bauchschmerz

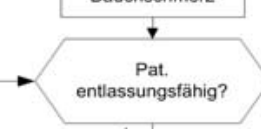

Ja $\downarrow$

Wiedervorstellung in 24-48 h

ggf. ambulante Abklärung

(Colo, Gastro, etc)

Abb.1 Klinischer Algorithmus für den Workflow bei akutem Abdominalschmerz. Der Algorithmus ist anzuwenden bei erwachsenen Patienten, die seit $<1$ Woche an abdominalen Schmerzen leiden und deshalb die Notfallambulanz aufsuchen.

${ }^{*}$ ) nach individueller Kontraindikation, Dosisanpassung nach Klinik und Pat.; ${ }^{* *}$ ) Pat. in klinischer Untersuchung hinreichend sicher beurteilt? 


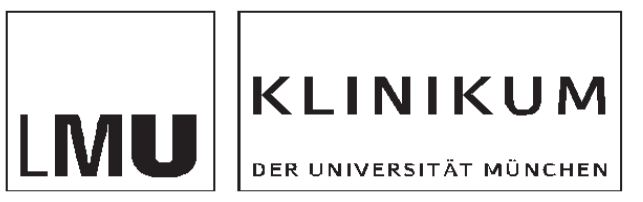

CAMPUS GROBHADERN

CHIRURGISCHE KLINIK UND POLIKLINIK DIREKTOR: PROF. DR. MED. K.W. JAUCH

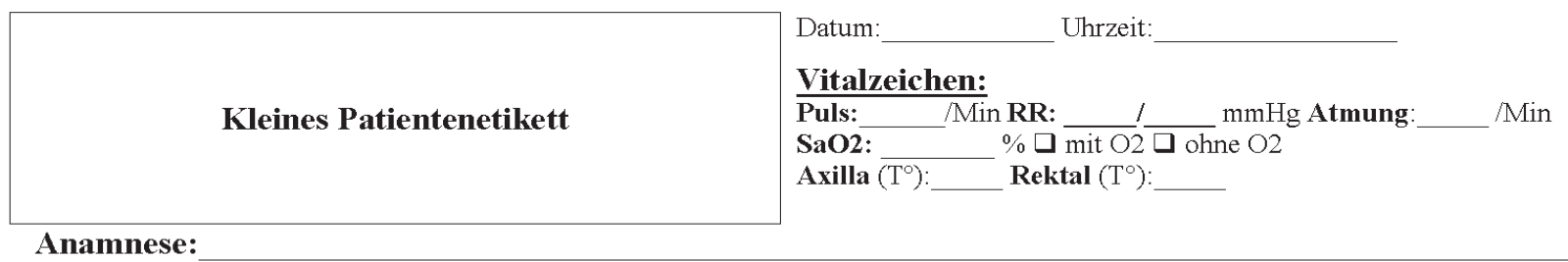

Erstereignis $\square \mathrm{Ja} \square \mathrm{Nein}$

Letzte Mahlzeit:

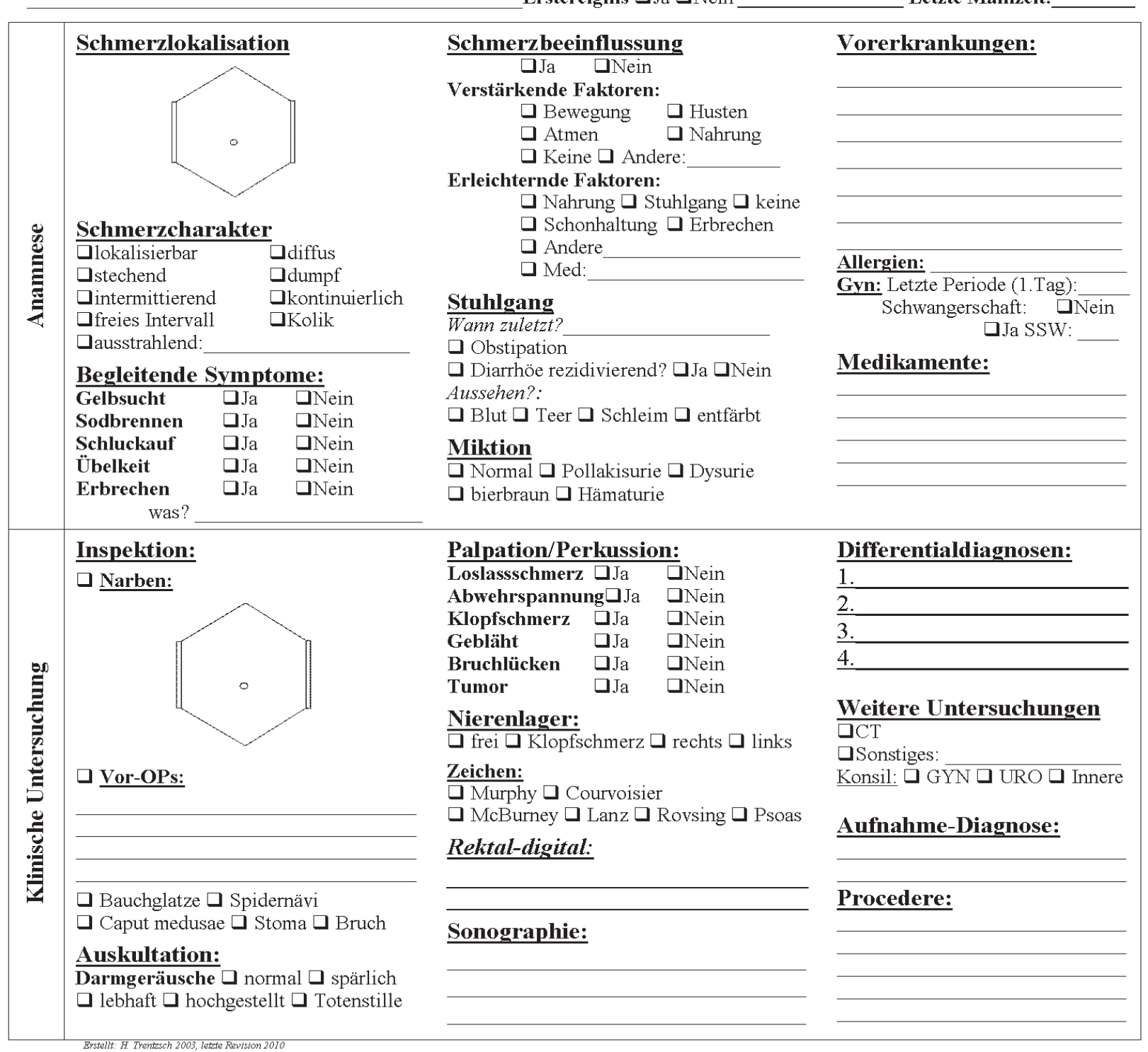

Untersucher:

Abb.2 Dokumentationsbogen „Akuter Abdominalschmerz”. Der Bogen dient zur standardisierten Dokumentation von Anamnese und Befund der körperlichen Untersuchung bei erwachsenen Patienten mit akutem Abdominalschmerz. Der Bogen basiert auf einem Vorschlag der OMGE [1]. 
Grundsätzlich sind von jedem Patienten bereits im Rahmen der Triage die Vitalparameter zu erheben. Für das akute Abdomen sollte jede Anomalität von Puls, Blutdruck, Atemfrequenz, Temperatur und Vigilanz sofort den Verdacht auf eine intraabdominale Katastrophe lenken. Brustschmerzen, Rückenschmerzen, Kurzatmigkeit, vaginale Blutung und hämodynamische Instabilität sind Warnsignale, die aber auch im Zusammenhang mit extra-abdominalen Ursachen stehen können und daher die Hinzuziehung von anderen Fachdisziplinen erfordern.

Das Leitsymptom des akuten Abdomens ist der Schmerz. Eine subtile Differenzierung kann wegweisend sein für die richtige Diagnose. Aufgrund der Art der afferenten Nervenbahn lassen sich drei Grundmuster benennen: Viszeraler Schmerz, somatischer Schmerz und übertragener Schmerz [5]:

Viszerale Schmerzempfindung geht typischer Weise auf Irritation der abdominalen Organe zurück, wenn diese Dehnung, Spannung, Verdrehung oder Kontraktion unterliegen. Der Schmerz wird über langsam-leitende C-Faser nach zentral geleitet. Der vom Patienten beschriebene Schmerz ist dumpf und schlecht lokalisierbar. Oft wird der Schmerz in der Medianlinie angegeben, weil die Innervation der Abdominalorgane in der Regel bilateral angelegt ist. Als Folge der embryonalen Darmentwicklung wird viszeraler Schmerz, der proximal des Treitz'schen Bandes (kaudaler Abschnitt des embryonalen Vorderdarms) entsteht (z. B. hepato-biliäres System oder Milz) im Epigastrium, zwischen Treitz'schem Band und rechter Kolonflexur (embryonaler Mitteldarm, Versorgungsgebiet der A. mesenterica superior) eher periumbilikal und viszeraler Schmerz distal der rechten Flexur (embryonaler Enddarm) eher im Unterbauch wahrgenommen.

Somatischer (parietaler) Schmerz entsteht direkt am Peritoneum und wird als scharf oder stechend beschrieben. Patienten können ihn deutlich besser lokalisieren. Die Leitung nach zentral erfolgt über schnellleitende A-delta-Fasern, die unilateral angelegt sind, was zu einer Lateralisierung des Schmerzes führt.

Übertragener Schmerz entsteht aus der Konvergenz von somatischen und viszeralen Afferenzen. Die Besonderheit dieses Schmerztyps ist es, dass der Schmerz nicht an der Stelle wahrgenommen wird, wo er entsteht, sondern sich in das korrespondierende, u.U. fernab lokalisierte Dermatom der somatischen Afferenz, die sog. Head'schen Zonen projiziert.

In Abhängigkeit von der Lokalisation der Schmerzwahrnehmung lassen sich, entsprechend Tab.1, oft schon frühzeitig typische Differenzialdiagnosen formulieren [14]. Allerdings können alle drei Schmerztypen nebeneinander vorkommen [5]!

Neben Schmerzcharakter und -lokalisation sowie begleitenden Symptomen und Faktoren, die die Beschwerden verstärken oder lindern, sollten auch Informationen über zeitliche Dynamik sowie Miktion, Defäkation und relevante Vorerkrankungen nebst stattgehabten Operationen abgefragt werden.

Die körperliche Untersuchung beginnt mit der sorgfältigen Inspektion, die sich nicht auf die Bauchdecke beschränken sollte. So werden z.B. Patienten mit einer Peritonitis in typischer Schonhaltung mit angezogenen Beinen regungslos auf der Liege ruhen, während Patienten mit Nierenkoliken mit motorischer Unruhe auftreten. Narben oder Stomata sind in der Regel leicht zu entdecken, manchmal sind Hernien durch die augenfällige Vorwölbungen des Bruchsackes schon bei bloßer Inspektion erkennbar und können genauso diagnoserelevant sein wie ein Sklerenikterus bei Cholestase.

Als nächster Schritt erfolgt die Auskultation, deren Stellenwert umstritten ist. Während das Fehlen von Darmgeräuschen eine normale Darmfunktion nicht ausschließen kann, sind lebhafte, ohrnahe („klingende“) und gurgelnde Darmgeräusche bei entsprechender Klinik verdächtig auf einen Ileus [14-16].

Die Palpation der Bauchdecke ist zur Abschätzung der Behandlungsdringlichkeit wahrscheinlich der wichtigste Untersuchungsteil. Eine grobe und zu tiefe Palpation kann jedoch selbst gesunden Patienten stärkste Schmerzäußerungen entlocken. Die Palpation sollte daher sanft erfolgen, ohne selbst schmerzhaft zu sein. Auf der anderen Seite kann die Tastuntersuchung insbesondere bei adipösen Patienten wenig hilfreich sein und das Fehlen eines Loslass-Schmerzes schließt eine floride Peritionitis auch nicht völlig aus. Ein guter Weg, Schmerzen aufgrund einer Peritonitis zu provozieren ist es, den Patienten husten zu lassen, am Bett anzustoßen oder die Bauchdecke einfach nur leicht zu beklopfen [5].

Es überrascht nicht, dass peritoneale Zeichen schlicht übersehen werden können. Dies gilt insbesondere für ältere Patienten mit wenig Bauchmuskulatur. Eine Analyse von 2121 forensischen Sektionen ergab in $5 \%$ ein nicht erkanntes akutes Abdomen als Todesursache bei Menschen > 70 Jahre. Hauptursache waren perforierte peptische Ulzera. Der Umstand, dass $62 \%$ der Patienten im Vorfeld einen Arzt aufsuchten und die Schlussfolgerung der Autoren, dass die frühzeitige Diagnosestellung diese Patienten wahrscheinlich hätte retten können, veranschaulicht in dramatischer Weise die Schwierigkeiten beim alten Patienten, das klassische Beschwerdebild zu erkennen [17]!

Die zur Komplettierung des Untersuchungsganges oft geforderte digital-rektale Untersuchung muss in ihrer Sinnhaftigkeit hinterfragt werden. Nach einer aktuellen Metaanalyse findet sich keine Evidenz für diese Untersuchung. Unabhängig davon besteht allerdings für die digital-rektale Untersuchung im Rahmen des Screenings nach Prostata- oder Rektumkarzinomen eine eindeutige Indikation (Werner JC, Zock M, Kanz K-G. Sinnhaftigkeit der digital rektalen Untersuchung bei akutem Abdomen in der Notfallaufnahme; Manuskript in Vorbereitung zur Publikation).

Angesichts der Tatsache, dass sich mindestens $1 / 3$ aller Patienten mit akutem Abdomen mit atypischen Beschwerden vorstellen [1], sind diagnostische Hilfsmittel wie Laboruntersuchungen und bildgebende Verfahren letztlich unverzichtbar. Schwerwiegende extraabdominale Ursachen wie Myokardinfarkt oder Laktatazidose bei Diabetes können nachhaltig die Prognose bei falsch-positiver OP-Indikation verschlechtern und sollten deshalb in jedem Fall ausgeschlossen werden [6]! Die Minimalanforderung für eine klinische Bewertung aus unserer Sicht umfasst daher eine laborchemische Basisuntersuchung sowie bei akutem Oberbauchschmerz ein 12-Kanal-EKG zum Ausschluss eines akuten Myokardinfarkts.

\section{Basislabor}

Der Umfang der Laboruntersuchung wird bestimmt durch die angenommene Verdachts- bzw. deren Differentialdiagnosen, sodass ggf. eine Erweiterung nach individueller Fragestellung nötig ist. Für eine orientierende Bewertung sollten folgende Parameter als Basislabordiagnostik verstanden werden: Kleines Blutbild, Creaktives Protein, Bilirubin, alkalische Phosphatase, Transaminasen (GOT/GPT) sowie $\gamma$-GT, Lipase / Amylase, Kreatinin, Kalium, Serumglukose und Laktat sowie ein U-Stix plus Sediment. Im Hinblick auf CT-Untersuchungen mit Kontrastmittel sollte ferner ein TSH bestimmt werden, bei Oberbauchschmerzen zum Ausschluss einer Mykardischämie ein Troponin. Bei Frauen im gebärfähigen Alter muss ein Schwangerschaftsausschluss mittels $\beta$ HCG-Test erfolgen. 


\section{Analgesie}

Generationen von Medizinern wurde in der Vergangenheit vermittelt, dem Patienten mit akutem Abdomen die Schmerztherapie solange vorzuenthalten, bis die endgültige Diagnose gestellt sei. Der Grund dafür war die Angst, richtungsweisende Symptome zu maskieren und den Befund der körperlichen Untersuchung zu verfälschen, was die ultimative Verzögerung der richtige Diagnose und somit eine Verzögerung von dringlich indizierten Therapiemaßnahmen wäre. Hier hat sich aber ein Paradigmenwechsel vollzogen. Eine Cochrane-Analyse [18] zeigt, dass durch eine adäquate Analgesie auf Opiatbasis der Patientenkomfort und somit die Qualität der Notfallversorgung steigt. Gleichzeitig wurde durch die Analgesie weder eine relevante Veränderung des Untersuchungsbefundes (Risk Ratio (RR) 1,23 [95\% CI 0,69, 2,20]) noch vermehrt Fehler bei der Diagnosestellung (RR 0,81 [95\% CI $0,48,1,37]$ ) oder der Therapieentscheidung (RR 0,77 [95\% CI 0,23, $2,54]$ ) beobachtet. Man sollte aber bedenken, dass vor Beginn der Analgesie der klinische Befund mit hinreichender Sicherheit beurteilt wurde!

In Anlehnung an den bei uns praktizierten klinikweiten Standard zur postoperativen Schmerztherapie [19] würden wir die Analgesie in Abhängigkeit der subjektiv empfundenen Schmerzintensität auf der Visuellen Analog-Skala (VAS) anpassen. Dabei kommen Opiate erst ab einer VAS von>3 zum Einsatz, z. B. 7,5 mg Piritramid i.v. bolusweise. Bei Schmerzen mit VAS $\leq 3$ kommen NichtOpiatanalgetika wie z.B. Acetaminophen $1 \mathrm{~g}$ i.v. oder Metamizol 2,5 g i. v. im Rahmen der individuellen Kontraindikationen in Betracht, bei Koliken erweitert um $10 \mathrm{mg}$ Buthyl-Scopolamin i.v.

\section{Chirurgische Taktik entsprechend der klinischen Präsentation \\ $\nabla$}

Angesichts der Vielzahl möglicher Erkrankungen, die dem Beschwerdebild zugrundeliegen, stellt sich im Anschluss an Anamnese und körperliche Untersuchung für den pragmatisch handelnden Chirurgen die Frage, ob eine Notwendigkeit zur operativen Therapie besteht und wann der richtige Zeitpunkt dafür ist. Es gilt die Möglichkeit einer intraabdominellen Katastrophe gegen eine harmlose, evtl. sogar nicht therapiebedürftige Ursachen abzuwägen. Während einerseits die Ursache des akuten Abdomens ein absolut lebensbedrohlicher Zustand sein kann, bei dem das Leben des Patienten ohne sofortige Operation sicher verloren ist, so kann man sich andererseits in ca. 2/3 der Fälle von einer operativen Intervention gänzlich verabschieden [2022].

Alleine aufgrund der klinischen Präsentation sind vier mögliche Vorgehensweisen vorstellbar: (1)Sofortige Operation, (2) präoperative Konditionierung und dringliche bzw. (früh-)elektive operative Therapie, (3) konservative Therapie unter stationären Bedingungen (aktive Observation) oder (4)Entlassung nach Hause zur Wiedervorstellung. Werden im Rahmen von Anamnese und körperlicher Untersuchung entsprechende klinische Muster entdeckt, so können sie die eine oder andere Vorgehensweise triggern [6]. Dabei sei betont, dass eine präzise Diagnose grundsätzlich der Schlüssel zur erfolgreichen Therapie bleibt, gerade wenn sie mit den Risiken eines operativen Eingriffes verbunden ist. Gemeint ist hier der Umstand, dass bei klinisch auffälligem Bauch, z.B. bei generalisierter Peritonitis mit der typischen Anamnese für ein peptisches Ulkus die Entscheidung zur OP schon vor der Bestätigung der Verdachtsdiagnose „Hohlorganperforation“ durch weiterführende Diagnostik gefällt wird. Diese chirurgische
Taktik wird von verschiedenen Autoren derart herausgestellt, dass sie unbedingt Eingang in die Überlegungen zu einem prioritätengesteuerten Workflow finden sollte $[1,6,12]$ :

Patienten mit starken abdominalen Schmerzen und kritischen Veränderungen der Vitalparameter bei gleichzeitig bestehenden Symptomen eines Schocks (blasses Hautkolorit, Kaltschweißigkeit, Hypothermie, Tachypnoe, Tachykardie mit Orthostase und Hypotension) sind höchst verdächtig auf Krankheitsbilder, die sofortiges operatives Handeln verlangen. Typische Beispiele sind das rupturierte Bauchaortenaneurysma oder die rupturierte, extrauterine Gravidität. Obwohl sehr selten, können sich diese Krankheitsbilder klinisch entsprechend drastisch darstellen und schon bei Verdacht im individuellen Fall eine sofortige OP ohne weitere Diagnostik erforderlich machen! Soweit der Zustand des Patienten es erlaubt, sollte selbstverständlich die Diagnostik ohne Zeitverlust und unter Einbindung des Schockraumteams so weit wie möglich komplettiert werden! Todesfälle im Rahmen von erweiterter Diagnostik bei Notfall-OP-Indikation sind beschrieben und erweiterte diagnostische Maßnahmen brachten in einer Serie von 300Fällen mit akutem Abdomen bei lebensbedrohlichen Ursachen nur bei jedem vierten Patienten zusätzlich nützliche Informationen; verzögerten aber in 30\% den Beginn der operativen Therapie [12].

Patienten, die sich mit zentralem, kolikartigen Abdominalschmerz, Distension, Verstopfung und / oder Erbrechen vorstellen, sind verdächtig für eine intestinale Obstruktion. Je ausgeprägter das Erbrechen, desto proximaler ist der Ort der Obstruktion; je distendierter das Abdomen, desto weiter distal ist der Ort der Obstruktion. Erbrechen und Koliken sind eher typisch für Dünndarmverschlüsse; Verstopfung, und deutliche Distension hingegen eher richtungsweisend für einen Dickdarmverschluss. Dieses Muster führt oftmals in Richtung operative Therapie, meist als frühelektiver Eingriff nach adäquater Vorbereitung des Patienten. Unkomplizierte Präsentation ohne Zeichen einer Peritonitis mit Operationen in der Anamnese rechtfertigen zunächst einen konservativen Ansatz. Bei Erbrechen oder Aufstoßen sollte zur Dekompression und als Aspirationsschutz eine Magensonde gelegt werden sowie eine adäquate intravenöse Flüssigkeitszufuhr erfolgen. Bei therapierefraktärer Obstruktion wird man sich im Verlauf für eine operative Vorgehensweise entscheiden. Bei Obstruktionen mit Zeichen einer Peritonitis, erhöhten Leukozyten und Fieber wird man hingegen frühzeitig operativ intervenieren. Weiterführende Diagnostik ist in beiden Fällen sicher sinnvoll. Lokalisierter Druck- und Loslass-Schmerz, beschränkt auf einen der vier Quadraten, begleitet von Schonhaltung und lokaler Abwehrspannung, sprechen für lokalen Peritonismus, der nicht zwangsläufig in einer OP enden muss. Gerade bei atypischer Präsentation ist es vorstellbar, zunächst abzuwarten. Je nach klinischem Bild sollte der Patient auf eine chirurgische Station zur aktiven Observation aufgenommen werden. Die Zeit ist dabei ein hervorragender Diagnostiker, der einem bald die fehlenden Hinweise gibt, die zur richtige Diagnose führen [6]. Die Indikation zum Einsatz bildgebender Verfahren stellt sich bei Zeichen der Sepsis oder Ischämie und bei zunehmender bzw. massiver Distension des Abdomens. Aus Befunden wie freier intraabdomineller Luft, Blutungszeichen (KM-Austritt) oder KM-Abbruch in der CT-Angio leitet sich die dringliche Indikation zur OP ab. Häufigste Vertreter dieses Musters sind im rechten unteren Quadranten die akute Appendizitis, im rechten oberen Quadranten die Cholezystitis und im linken unteren Quadranten die Divertikulitis. Für das Timing der operativen Therapie bei diesen Krankheitsbildern existiert eine Reihe von Therapieempfehlungen [23], dennoch 
bleibt die Entscheidung für die frühelektive oder gar elektive Planung des Eingriffes eine Herausforderung für den Chirurgen, bei der Überlegungen hinsichtlich Ressourcen und Kapazitäten nicht hinten angestellt werden sollten.

Patienten mit diffusem Abdominalschmerz, Schonhaltung, bretthartem Abdomen, Loslassschmerz und Abwehrspannung sind verdächtig für eine generalisierte Peritionitis. Wenn begleitet von den typischen Zeichen einer Sepsis (besteht nach der Definition durch die ACCP/SCCM Consensus Conference [24] bei angenommener oder bestätigter Infektion und dem Vorliegen von mindestens 2 Kriterien für ein Systemisches Inflammatorisches Response Syndrom [SIRS]). SIRS-Kriterien sind: Körpertemperatur $\leq 36^{\circ} \mathrm{C}$ oder $\geq 38^{\circ} \mathrm{C}$, Leukozytose $\geq 12000 / \mathrm{mm}^{3}$ oder Leukopenie $\leq 4000 / \mathrm{mm}^{3}$ oder $\geq 10 \%$ stabförmige Granulozyten, Tachykardie mit Herzfrequenz $\geq 90 / \mathrm{min}$, Tachypnoe mit Atemfrequenz $>20 /$ min oder $\mathrm{PaCO}_{2} \leq 4,3 \mathrm{kPa}$ bzw. $\leq 33 \mathrm{mmHg}$ ) oder begleitet von Ischämiezeichen (Fieber, Tachykardie, Hypotension, Laktatanstieg) stellt sich die Indikation zur dringlichen OP. Typische Krankheitsbilder sind Ulkus- oder Kolonperforationen sowie die perforierte Appendizitis. Die akute Pankreatitis stellt hier eine wichtige Ausnahme dar, denn obwohl im Vordergrund der initialen Phase eine massive, systemische Entzündungsreaktion steht, beschränkt sich deren Management zunächst auf ein konservatives Vorgehen [25], weswegen eine zusätzliche Bildgebung im individuellen Fall indiziert ist.

Auf der Suche nach operativ angehbaren Ursachen sollten differenzialdiagnostisch auch die extraabdominellen Ursachen nicht vergessen werden. Dabei wird üblicher Weise bei allen weiblichen Patienten der Gynäkologe hinzugezogen, bei bekannter Schwangerschaft oder positivem $\beta$-HCG-Test u.U. auch schon sehr früh im zeitlichen Ablauf des Workflows [26, 27]. Neben internistischen Ursachen sollte aufgrund der kritischen zeitlichen Dynamik und den möglichen medikolegalen Konsequenzen bei männlichen Patienten als Differenzialdiagnose ein akutes Skrotum z.B. durch eine Hodentorsion erwogen werden. Gerade bei Erwachsenen und atypischen Beschwerden kann die sonst augenfällige Diagnose verschleiert sein. Bei akutem Skrotum muss eine urologische Notfall-Konsultation erfolgen [28, 29].

\section{Erweiterte diagnostische Maßnahmen: Bildgebung}

Die bildgebende Diagnostik nimmt in der Evaluierung unstrittig eine zentrale Rolle ein [30]. Obwohl der konventionellen Röntgenaufnahme des Abdomens im Stehen oder in Linksseitenlage von Praktikern durchaus ein besonderer Stellenwert eingeräumt wird [31], gilt sie heute als ein von Sonografie und CT abgelöstes Verfahren [13, 32]. In einer Studie, die sich dem Management von Patienten mit Indikation zur Notfall-OP widmet, sehen Rozycki et al. in der Leeraufnahme bei Patienten mit V.a. Hohlorganperforation jedoch eine Rechtfertigung, um bei Nachweis von freier Luft zugunsten der umgehenden operativen Therapie auf weiterführende Diagnostik zu verzichten [12].

Die abdominale Sonografie ist in der Hand des Erfahrenen hingegen eine exzellente Methode zur diagnostischen Evaluierung des akuten Abdomens. Als besonderer Vorteil der Methode gilt, dass die Untersuchung dynamisch und in Echtzeit erfolgt. Dadurch können nicht nur Peristaltik des Darms oder Perfusion von Gefäßen beurteilt werden, es gibt auch die Möglichkeit, das Schmerzzentrum einer gezielten Evaluierung zu unterziehen [30]. Ultraschall ist nicht-invasiv, beliebig wiederholbar und in der Regel überall verfügbar. Auf der anderen Seite gilt Ultraschall bezüglich der Präzision als stark untersucherabhängig, wodurch die Aussagekraft geschmälert wird [13]. Insbesondere bei Notfallpatienten kann die Beurteilbarkeit auch für erfahrene Anwender beeinträchtigt sein, da die Patienten zum Zeitpunkt der Untersuchung in der Regel nicht vorbereitet sind, also keine Nahrungskarenz hinter sich oder z.B. bei Darmobstruktion intraluminale Luftansammlung haben, die zu Auslöschungsphänomenen führen. Dennoch sollte aus unserer Sicht die Sonografie als weiterführende Diagnostik an erster Stelle stehen.

Eine wesentlich objektivere und gut reproduzierbare Methode mit geringer Interobserver-Variabilität ist die Computertomografie (CT) [33]. Sie ist jedoch deutlich teurer und geht mit einer erheblichen Strahlenbelastung für den Patienten einher. Ihr diagnostischer Wert auf der anderen Seite ist unbestritten [30]. Zur Evaluierung des akuten Abdomens ist die CT mit i.v.-Kontrastierung etabliert. Zusätzlich kann sie mit oraler und rektaler Kontrastmittelgabe kombiniert werden, was die diagnostische Treffsicherheit in der Mehrzahl der Fälle nicht wesentlich erhöht, den Aufenthalt des Patienten in der Notaufnahme aber verlängert [30]. In einer retrospektiven Analyse von 2222 Patienten mit akutem Bauchschmerz konnte die Diagnose in 96,8\% mittels CT gestellt werden. Durch den Einsatz des CT konnte durch frühzeitige und korrekte Diagnose auch eine deutliche Reduktion der stationären Aufnahmen bzw. eine Verkürzung des Krankenhausaufenthaltes erreicht werden [34]. Des Weiteren sollte die Möglichkeit einer diagnostisch-therapeutischen Intervention, z. B. der perkutanen Anlage von Dränagen nicht unterschätzt werden.

Neben den hohen Kosten in Vorhaltung und Durchführung der CT besteht ein klarer Nachteil in der damit verbundenen Strahlenexposition und dem dadurch erhöhten Lebenszeitrisiko für eine strahleninduzierte Krebserkrankung mit tödlichem Ausgang. Dieses Risiko steigt umgekehrt proportional zum Alter des Patienten an. Die Strahlendosis einer abdominalen CT beträgt je nach Gerät und Protokoll bis zu 10 mSv. Für einen 25-jährigen Patienten ergibt sich daraus ein Risiko für eine Strahlen-induzierte Krebserkrankung von 1:900 und für eine Krebserkrankung mit tödlichem Ausgang von 1:1800; für einen 50-jährigen Patienten ein Risiko von $1: 1500$ bzw. $1: 2500$. Damit ist klar, dass nicht nur aus ökonomischen Gründen, sondern auch hinsichtlich der Patientensicherheit die Indikation zur CT streng zu stellen ist $[13,35]$.

Die Magnet-Resonanz-Tomografie (MRT) hat in der Routineabklärung des akuten Abdomens kaum einen Stellenwert. Obwohl die Bildqualität hervorragend ist, ist der Einsatz dieser Methode durch den Umstand begrenzt, dass die Untersuchungen zeitaufwändig sind und Geräte nicht überall und selbst in großen Zentren nicht immer rund um die Uhr verfügbar sind [30].

Unter Abwägung von Vor- und Nachteilen der verschiedene Methoden zur bildgebenden Diagnostik sehen wir ein Vorgehen, basierend auf den Daten der OPTIMA-Studie [13]. Diese prospektiv angelegte Multicenterstudie an einer konsekutive Serie von 1101 Patienten mit akutem Abdomen prüfte die Präzision verschiedener diagnostischer Strategien. Unter der Maßgabe, bildgebende Diagnostik kostenbewusst und unter Würdigung des Strahlenschutzes sinnvoll einzusetzen, wurde eine konditionale Strategie erarbeitet, bei der jeder Patient mit akutem Abdomen neben der klinischen Untersuchung einer abdominalen Sonografie unterzogen wurde. Bei allen negativen oder unklaren Befunden schloss sich eine CT mit i.v.-Kontrast an, wodurch eine Sensitivität von $94 \%$ und eine Spezifität von 68\% erzielt wurde (positive Likelihood-Ratio 2,94). Mit dieser Vorgehensweise wurden Diagnosen, die innerhalb der ersten $24 \mathrm{~h}$ eine dringliche therapeutische Intervention erfordert hätten, in nur 6\% der Fälle 
übersehen. Gleichzeitig war ein CT-Einsatz aber nur in 49\% der Fälle erforderlich, was zu einer deutlichen Strahlenreduktion führte. Damit war diese Strategie allen anderen Vorgehensweisen des Studienprotokolls überlegen. Höhere Likelihood-Ratios von 3,87 bzw. 4,05 wurden nur durch Strategien erzielt, die einen CT-Einsatz in 100 bzw. 95\% der Fälle erforderlich machten, aber in $11 \%$ der Fälle eine Diagnose mit dringlicher Therapieindikation übersahen! Hierzu zählten patientenzentrierte Strategien, die die Schmerzlokalisation oder den Body-Mass-Index berücksichtigten [13].

Schlussfolgernd sollten daher alle Patienten mit V.a. akutes Abdomen, nach umfassender Anamnese und sorgfältiger körperlicher Untersuchung einer abdominalen Sonografie unterzogen werden. Ergibt sich hieraus eine klare Ursache, ist eine entsprechende chirurgische Therapie gemäß der gebotenen Dringlichkeit einzuleiten. Beispiele für solche sprichwörtlichen „Augenscheindiagnosen" sind die akute Cholezystitis, die inkarzerierte Leistenhernie oder die typische Appendizitis. Bei allen Patienten, bei denen die Ursache unklar bleibt, die aber weder Auffälligkeiten in der körperlichen Untersuchung noch im Labor zeigen und bei denen darüber hinaus kein Risikofaktor für eine chirurgische Ursache ihrer Beschwerden vorliegt, kann aus epidemiologischer Sicht mit hoher Wahrscheinlichkeit ein nicht-spezifischer Bauchschmerz angenommen werden [1]. Risikofaktoren für chirurgisch angehbare Ursachen wurden in einer Studie an 1000 konsekutiven Patienten gefunden: (1) Schmerzen seit weniger als $48 \mathrm{~h}$; (2) Schmerz nach Erbrechen; (3) Abwehrspannung und Loslassschmerz bei der Tastuntersuchung; (4) fortgeschrittenes Alter ( $\geq 65$ Jahre); (5) Voroperation in der Krankengeschichte [21]. Sind bei ansonsten unauffällige körperliche Untersuchungsbefund solche Risikofaktoren oder gravierende Auffälligkeiten im Basislabor vorhanden, so sollte die Indikation zur CT großzügig gestellt werden. Gerade bei älteren Patienten mit akutem Abdomen schließt eine unspektakuläre klinische Präsentation eine signifikante Ursache nicht aus. Mortalität und Morbidität in dieser Gruppe sind hoch. So berichtet eine Studie an 380 Patienten $>65$ Jahre mit akutem Abdomen, dass $5 \%$ an den Folgen der auslösenden Ursache verstarben und $22 \%$ einer operativen Therapie bedurften [36]. Der Einsatz der Bildgebung mit CT sollte entsprechend liberal erfolgen [37].

\section{Konservatives Vorgehen: Aktive Observation oder Wiedervorstellung im Verlauf? \\ $\nabla$}

Bleibt die Ursache der Beschwerden unklar und lassen sich keine objektiven Befunde erheben, die ein operatives Vorgehen rechtfertigen, kann die konservative Therapie gewählt werden. Aus epidemiologischer Betrachtung wird man für ein Drittel der Fälle den V.a. auf nicht-spezifischen Bauchschmerz äußern. Es stehen zwei mögliche Optionen zur Verfügung: Entlassung nach Hause oder stationäre Aufnahme.

Der Vorteil der stationären Aufnahme liegt in der Möglichkeit zur aktiven Observation, bei der idealer Weise durch ein und denselben Arzt eine klinische Verlaufskontrolle, u.U. in kurzem zeitlichen Intervall möglich ist. Gerade bei Patienten mit starken Schmerzen, begleitet von Flüssigkeitsdefiziten, Elektrolytstörungen oder erhöhten Entzündungszeichen, eignet sich dieser Weg, da er neben kontinuierlicher Vitalfunktionskontrolle auch symptomatische Therapien wie Flüssigkeitssubstitution, abführende Maßnahmen, Analgesie oder ggf. eine Antibiotikatherapie erlaubt.
Lassen Symptome, Intensität der Beschwerden, Einsichtsfähigkeit und Complinance des Patienten dies zu, dann kann alternativ auch die Entlassung erwogen werden, wenn gewährleistet ist, dass der Patient über Telekommunikationsmöglichkeiten zum Hinzuziehen von Hilfe verfügt oder sich vor Ort betreuende Personen befinden, kann bei vernünftiger Entfernung zur nächsten medizinischen Einrichtung eine Wiedervorstellung bei Verschlechterung oder innerhalb eines vorher festgelegten Intervalls erfolgen.

Die Befürchtung, dass durch ein solches Abwarten möglichen Komplikationen Tür und Tor geöffnet wird, ließ sich in Studien nicht belegen. An 500 Patienten zeigte sich bei Wiedervorstellung nach $24 \mathrm{~h}$ zwar in $30 \%$ eine Änderung der initialen Diagnose und in $17 \%$ eine Änderung der Therapie einschließlich einer OP-Rate von $4 \%$, die aber nur in $1,2 \%$ im Sinne einer Therapieverzögerung gewertet wurden und die ohne Einfluss auf die Morbidität blieb [38].

Eine andere Studie zeigt, dass nach Aufnahme zur aktiven Observation nicht mehr Komplikationen beobachtet werden, dafür aber die Rate der negativen Laparotomien deutlich absinkt [39]. Dieses Vorgehen ist nicht nur sicher, sondern erhöht auch die Patientensicherheit.

Bei Wiedervorstellung des Patienten nach $24-48 \mathrm{~h}$ sollte eine sorgfältige klinische Reevaluierung und Laborkontrolle erfolgen. Sind die Beschwerden gebessert oder verschwunden ist unter Umständen keine weitere Therapie erforderlich. Es sollte aber bedacht werden, dass bei allen Patienten, die sich mit unklarem Abdominalschmerz vorstellen, ohne dass eine triftige Ursache gefunden wird, weiter ambulant oder im niedergelassenen Bereich abgeklärt wird, da sich hinter vermeidlich unspezifischen Bauchschmerzen gerade mit zunehmendem Alter maligne Erkrankungen verbergen können [1].

\section{Invasive Diagnostik}

$\nabla$

Eine Ausdehnung des diagnostischen Aufwandes stellen invasive Untersuchungsmethoden dar. Einen hohen Stellenwert haben hier die endoluminalen Verfahren am oberen und unteren Gastrointestinaltrakt, weil sie eine Brücke zwischen Diagnostik und Intervention darstellen. Endoskopische Untersuchungen, die in der Regel eine Vorbereitung des Patienten und Aufklärungsformalitäten erfordern, stehen im Rahmen der Notfallevaluierung allerdings meist nicht zur Verfügung [7].

Nach Ausschöpfung aller nicht-invasiven diagnostischen Maßnahmen kann in Anlehnung an die Leitlinien der Society of American Gastrointestinal Surgeons (SAGES) die diagnostische Laparoskopie in Erwägung gezogen werden (GradC: „Kann“-Empfehlung). Ob dieses Vorgehen zur Abklärung eines nicht-spezifischen Bauchschmerzes der aktiven Observation vorzuziehen ist, kann angesichts unzureichender Evidenz nicht gesagt werden [40]. Die bei laparoskopischer Evaluierung am häufigsten durchgeführten Eingriffe sind Appendektomien (31\%), Cholezystektomien (19\%) und gynäkologische Eingriffe am Ovar [41]. Eine Metaanalyse [42], die die Frage nach explorativer Laparoskopie vs. aktiver Observation bei V.a. nicht-spezifischen Bauchschmerz beleuchtet, folgert, dass es keine ausreichende Evidenz gibt, die die diagnostische Laparoskopie als Goldstandard empfehlen kann, andererseits sich aber auch kein Anhalt dafür besteht, dass durch dieses Vorgehen ein Nachteil für den Patienten entsteht. Insbesondere bei Frauen mit Unterbauchbeschwerden wird in der SAGES-Leitlinie eine großzügige Indikation zur Laparoskopie 
empfohlen [40]. Auf der anderen Seite gibt es Hinweise aus einer prospektiv-randomisierten Studie an 53Frauen im Alter zwischen 13 bis 45Jahren mit V.a. nicht-spezifischen Abdominalschmerz, dass sich kein Nachteil ergibt, wenn die Laparoskopie um 24 bis 72 h aufgeschoben wird, obwohl fast $40 \%$ der Observationsgruppe im Verlauf eine operative Therapie wegen Symptomverschlechterung erhielt. Dem gegenüber stand eine bessere diagnostische Genauigkeit der explorativen Laparoskopie, bei der in lediglich $17 \%$ der Fälle keine eindeutige Diagnose gestellt werden konnte, während in der Beobachtungsgruppe die Ursache in $55 \%$ unklar blieb [43].

\section{Diskussion}

\section{$\nabla$}

Hochkomplexe Behandlungsabläufe können durch klinische Algorithmen in einen übersichtlichen, logisch koordinierten und systematischen Gesamtprozess umgesetzt werden [8]. Sie repräsentieren wissenschaftlich anerkannte Standards, zeigen einen strukturierten Lösungsweg auf und machen Zusammenhänge verständlich. Sie bilden eine einheitliche und allgemein gültige Behandlungsvorschrift, gestatten aber in begründeten Fällen Abweichungen. Ihre Anwendung ermöglicht eine systematische Fehleranalyse bei qualitätssichernden Maßnahmen. In Notfallsituationen zeigen sie dem unerfahrenen Anwender einen strukturierten Lösungsweg und vermitteln dadurch trotz Zeitdruck Sicherheit. Algorithmen dienen als Lehrmaterial in Aus- und Weiterbildung und stellen die Basis jedes Qualitätsmanagements dar, da sie den Soll-Prozessablauf vorgeben, anhand dessen der Ist-Zustand gemessen werden kann $[8,10]$. Klinische Algorithmen bilden Entscheidungsabläufe und Problemlösungen nach fest definierten Regeln ab. Die Darstellung des Entscheidungsablaufes erfolgt hierbei durch Flussdiagramme, die durch eindeutige Ja / Nein-Kriterien einer binären Logik folgen [10].

Wir haben für die Evaluierung von erwachsenen Patienten, die wegen akuter Abdominalschmerzen die Nothilfe aufsuchen, nach einem problem- und prioritätenorientierten Algorithmus gefragt, der einen strukturierten Lösungsweg vorgibt, um eine gezielte Entscheidungsfindung für die diagnostische Aufarbeitung und die korrekten Therapieentscheidungen unter Berücksichtigung von typischen Fallstricken zu erreichen. Nach Durchsicht der verfügbaren Literatur zeichnet sich ab, dass ein solcher Algorithmus, der die genannten, formalen Kriterien erfüllt [9, 10], bisher nicht verfügbar ist.

Die genaue Prozessanalyse ist die intellektuelle Grundlage für die Formulierung eines Algorithmus. Waydhas et al. fordern daher, dass man die innere Struktur des medizinischen Entscheidungsprozesses und Handlungsablaufes, der in einem Algorithmus beschrieben werden soll, in möglichst klarer und geradliniger Weise offenlegt [10]. Aus diesem Grund haben wir, basierend auf den Eindrücken der täglichen Praxis, einen Workflow formuliert, der eine schlichte Aneinanderreihung der Einzelprozesse und der zu treffenden Entscheidungen sammelt, wobei wir versucht haben, eine Reihe wissenschaftlich gewonnener Empfehlungen und Studiendaten einzubeziehen. In erste Linie soll die Arbeit die Probleme aufdecken, die es bei der Entwicklung dieses klinischen Algorithmus zu lösen gilt. Sie bietet aber auch erste Lösungen an. Auf diese Weise beschreiben wir eine mögliches Konzept, dass im Detail nach Kriterien der evidenzbasierten Medizin weiter ausgebaut werden muss, um als methodische Grundlage für eine Behandlungsrichtlinie dienen zu können.
Eine systemimmanente Schwäche unseres Ansatzes ist, dass ein Algorithmus für dieses hochkomplexe Problem nicht in der Lage ist, Sonderfälle oder besondere Konstellationen zu berücksichtigen. Er soll Lösungsstrategien bieten, die für die Mehrzahl der Fälle richtig ist. Eine Überprüfung, ob dies tatsächlich erreicht werden kann, müsste dann in einer prospektiven klinischen Erprobung stattfinden. Des Weiteren darf man den Algorithmus nicht als unabänderliches Diktat missverstehen.

Angesichts der umfangreichen Literatur zu diesem Thema und der Komplexität des klinischen Problems kann kein Anspruch auf Omnipotenz erhoben werden. So lässt der Algorithmus die spezifische Diagnose von dezidiert benannten Krankheitsbildern oder die Entscheidung für das geeignetste chirurgische Therapieverfahren unberücksichtigt. Im Fokus des Algorithmus steht die Festlegung der Behandlungspriorität und der damit verbundene diagnostische Aufwand.

\section{Zusammenfassung}

Klinische Algorithmen dienen dazu, den Umgang mit hochkomplexen Krankheitsbildern wie dem Symptomkomplex des akuten Bauchschmerzes in einen übersichtlichen, logisch koordinierten und systematischen Gesamtprozess zu setzen. Ein so formulierter Prozess dient nicht nur als standardisierte Behandlungsrichtlinie mit dem Anspruch der bestmöglichen medizinischen Versorgung für die Mehrheit der Fälle, sondern erfüllt darüber hinaus Beiträge zur Qualitätssicherung, zur Aus- und Weiterbildung und zur Patientensicherheit. Für den akuten Bauchschmerz ist der umfassende Stand der Wissenschaft bisher nicht in einem solchen Algorithmus entsprechend der formalen Anforderungen niedergelegt. Wir haben mit dieser Arbeit einen ersten Schritt für die Erstellung eines solchen evidenzbasierten Algorithmus geliefert, indem wir die Einzelprozesse und die Rahmenbedingungen, die in dem Algorithmus berücksichtig werden müssen, beschrieben haben.

\section{Danksagung \\ $\nabla$}

Besonderer Dank gilt Herrn PD Dr. K.-G. Kanz für die Heranführung an das spannende Thema „Klinische Algorithmen“ und Herrn Prof. Dr. W. Hartl für seine Unterstützung bei der Literaturrecherche.

\section{Interessenkonflikt: Nein}

\section{Literatur}

1 Delcore R, Cheung LY. Acute Abdominal Pain. In: Souba WW, Mitchell FP, Jurkovich GJ Hrsg.. ACS Surgery Principles \& Practice. New York: WebMD; 2004: 253-268

2 Irvin TT. Abdominal pain: a surgical audit of 1190 emergency admissions. Br J Surg 1989; 76: 1121-1125

3 de Dombal FT. The OMGE acute abdominal pain survey. Progress report, 1986. Scand J Gastroenterol Suppl 1988; 144: 35-42

4 Lankisch PG, Mahlke R, Lübbers H. Das akute Abdomen aus internistischer Sicht. Dtsch Arztebl 2006; 103: A2179-A2188

5 Flasar MH, Goldberg E. Acute abdominal pain. Med Clin North Am 2006; 90: 481-503

6 Schein M. The Acute Abdomen. In: Schein M, Rogers PN Hrsg. Schein's Common Sense Emergency Abdominal Surgery; 2nd edition Aufl. Berlin, Heidelberg: Springer; 2005: 19-25

7 Grundmann RT, Petersen M, Lippert $\mathrm{H}$ et al. [The acute (surgical) abdomen - epidemiology, diagnosis and general principles of management]. Z Gastroenterol 2010; 48: 696-706 
8 Kanz KG, Sturm JA, Mutschler W. [Algorithm for prehospital blunt trauma management]. Unfallchirurg 2002; 105: 1007-1014

9 Khalil PN, Kleespies A, Angele MK et al. The formal requirements of algorithms and their implications in clinical medicine and quality management. Langenbecks Arch Surg 2011; 396: 31-40

10 Waydhas C, Kanz KG, Ruchholtz S et al. [Algorithms in trauma management]. Unfallchirurg 1997; 100: 913-921

11 Cartwright SL, Knudson MP. Evaluation of acute abdominal pain in adults. Am Fam Physician 2008; 77: 971-978

12 Rozycki GS, Tremblay L, Feliciano DV et al. Three hundred consecutive emergent celiotomies in general surgery patients: influence of advanced diagnostic imaging techniques and procedures on diagnosis. Ann Surg 2002; 235: 681-688 discussion: 688-689

13 Laméris W, van Randen A, van Es HW et al. Imaging strategies for detection of urgent conditions in patients with acute abdominal pain: diagnostic accuracy study. BMJ 2009; 338: b2431

14 Yamamoto $W$, Kono H, Maekawa $M$ et al. The relationship between abdominal pain regions and specific diseases: an epidemiologic approach to clinical practice. J Epidemiol 1997; 7: 27-32

15 Gade J, Kruse P, Andersen OT et al. Physicians' abdominal auscultation. A multi-rater agreement study. Scand J Gastroenterol 1998; 33: 773-777

16 Madsen D, Sebolt T, Cullen L et al. Listening to bowel sounds: an evidence-based practice project: nurses find that a traditional practice isn't the best indicator of returning gastrointestinal motility in patients who've undergone abdominal surgery. Am J Nurs 2005; 105 : 40-49 quiz: 49-50

$17 \mathrm{Ng} \mathrm{CY}$, Squires TJ, Busuttil A. Acute abdomen as a cause of death in sudden, unexpected deaths in the elderly. Scott Med J 2007; 52: 20-23

18 Manterola C, Astudillo $P$, Losada $\mathrm{H}$ et al. Analgesia in patients with acute abdominal pain. Cochrane Database Syst Rev CD005660 DOI: 10.1002 | 14651858.CD005660.pub2 2007

19 Mirow C. Schmerzmanagement im Krankenhaus - Evaluation der Schmerzintensität bei postoperativen Patienten und deren Veränderung nach Einführung eines Schmerztherapiestandards [Dissertation]. München: Ludwig-Maximilians-Universität; 2009: 63-64

20 Purcell TB. Nonsurgical and extraperitoneal causes of abdominal pain. Emerg Med Clin North Am 1989; 7: 721-740

21 Brewer BJ, Golden GT, Hitch DC et al. Abdominal pain. An analysis of 1000 consecutive cases in a University Hospital emergency room. Am J Surg 1976; 131: 219-223

22 Hawthorn IE. Abdominal pain as a cause of acute admission to hospital. J R Coll Surg Edinb 1992; 37: 389-393

23 Green JM. When is faster better? Operative timing in acute care surgery. Curr Opin Crit Care 2008; 14: 423-427

24 Bone RC, Balk RA, Cerra FB et al The ACCP/SCCM Consensus Conference Committee. American College of Chest Physicians/Society of Critical Care Medicine. Definitions for sepsis and organ failure and guidelines for the use of innovative therapies in sepsis. Chest 1992; 101: 1644-1655

25 Strobel O, Uhl W, Müller CA et al. Akute Pankreatitis - intensivmedizinische oder chirurgische Therapie? Chir Gastroenterol 2002; 18: 216222
26 Augustin G, Majerovic M. Non-obstetrical acute abdomen during pregnancy. Eur J Obstet Gynecol Reprod Biol 2007; 131: 4-12

27 Sharp HT. The acute abdomen during pregnancy. Clin Obstet Gynecol 2002; 45: 405-413

28 Henning J, Waxman S. Legal aspects of men's genitourinary health. Int J Impot Res 2009; 21: 165-170

29 Matteson JR, Stock JA, Hanna MK et al. Medicolegal aspects of testicular torsion. Urology 2001; 57: 783-786 discussion: 786-787

30 Stoker J, van Randen A, Lameris Wet al. Imaging patients with acute abdominal pain. Radiology 2009; 253: 31-46

31 Schein M. Abdominal Imaging. In:Schein M, Rogers PN Hrsg.. Schein's Common Sense Emergency Abdominal Surgery.; 2nd edition. Aufl. Berlin, Heidelberg: Springer; 2005: 33-54

32 Smith JE, Hall EJ. The use of plain abdominal x rays in the emergency department. Emerg Med J 2009; 26: 160-163

33 van Randen A, Lameris W, Nio CY et al. Inter-observer agreement for abdominal CT in unselected patients with acute abdominal pain. Eur Radiol 2009; 19: 1394-1407

34 Stromberg C, Johansson G, Adolfsson A. Acute abdominal pain: diagnostic impact of immediate CT scanning. World J Surg 2007; 31: $2347-$ 2354 discussion: 2355-2348

35 [Anonym]. The 2007 Recommendations of the International Commission on Radiological Protection. ICRP publication 103. Ann ICRP 2007; 37: 1-332

36 Marco CA, Schoenfeld CN, Keyl PM et al. Abdominal pain in geriatric emergency patients: variables associated with adverse outcomes. Acad Emerg Med 1998; 5: 1163-1168

37 Lewis LM, Banet GA, Blanda $M$ et al. Etiology and clinical course of abdominal pain in senior patients: a prospective, multicenter study. J Gerontol A Biol Sci Med Sci 2005; 60: 1071-1076

38 Toorenvliet BR, Bakker RF, Flu HC et al. Standard outpatient re-evaluation for patients not admitted to the hospital after emergency department evaluation for acute abdominal pain. World J Surg 2010; 34: 480-486

39 Thomson HJ, Jones PF. Active observation in acute abdominal pain. Am J Surg 1986; 152: 522-525

40 Society of American Gastrointestinal and Endoscopic Surgeons (SAGES), Guidelines for Diagnostic Laparoscopy (11.2007). Im Internet: www. sages.org/publication/id/12/; Stand: 10.03 .2011

41 Ahmad TA, Shelbaya E, Razek SA et al. Experience of laparoscopic management in 100 patients with acute abdomen. Hepatogastroenterology $2001 ; 48: 733-736$

42 Maggio AQ Reece-Smith AM, Tang TY et al. Early laparoscopy versus active observation in acute abdominal pain: systematic review and meta-analysis. Int J Surg 2008; 6: 400-403

43 Morino M, Pellegrino L, Castagna E et al. Acute nonspecific abdominal pain: A randomized, controlled trial comparing early laparoscopy versus clinical observation. Ann Surg 2006; 244: 881-886 discussion: 886-888 